vivo and in vitro demonstrates that $\mathrm{Ca}_{v} 1.3$ channels constitute the major component of the L-type current in pacemaker cells (8, 11, 12). Experimental results presented by Sinnegger-Brauns and coworkers clearly support this view (2). More generally, the use of engineered mice such as $\mathrm{Ca}_{V} 1.2 \mathrm{DHP}^{-/-}$mice will be of particular interest to further assess the contribution of the different ionic currents underlying diastolic depolarization in sinoatrial pacemaker cells. Automaticity in cardiac pacemaker cells is due to the slow diastolic depolarization phase, which drives the membrane voltage from the end of the action potential to the threshold of the following action potential. Both native and recombinant $\mathrm{Ca}_{v} 1.3$ channels exhibit more negative threshold for activation and slower inactivation kinetics than $\mathrm{Ca}_{v} 1.2$ channels. In other words, the low threshold of $\mathrm{Ca}_{v} 1.3$ current is consistent with a major role during diastolic depolarization (12), while a sustained calcium influx is required for the contractility of heart cells.

In conclusion, the originality of this model offers stimulating prospects for dissecting the physiological roles of calcium channels in various tissues (2). This genetic "reverse" pharmacology in vivo is likely to be applied in the future to other channels and receptor families sharing a similar pharmacology.

Address correspondence to: Emmanuel Bourinet, Département de Physiologie, Laboratoire de Génomique Fonctionnelle, Centre National de la Recherche Scientifique Unité Propre de Recherche 2580, 141, rue de la Cardonille, 34396 Montpellier Cedex 05, France. Phone: 33-499-61-99-36; Fax: 33-499-61-99-01; E-mail: Emmanuel. Bourinet@igh.cnrs.fr.

1. Striessnig, J. 1999. Pharmacology, structure and function of cardiac L-type $\mathrm{Ca}(2+)$ channels. Cell. Physiol. Biochem. 9:242-269.

2. Sinnegger-Brauns, M.J., et al. 2004. Isoform-specific regulation of mood behavior and pancreatic $\beta$ cell and cardiovascular function by L-type $\mathrm{Ca}^{2+}$ channels. J. Clin. Invest. 113:1430-1439. doi:10.1172/JCI200420208.

3. Tanabe, T., Beam, K.G., Powell, J.A., and Numa, S. 1988. Restoration of excitation-contraction coupling and slow calcium current in dysgenic muscle by dihydropyridine receptor complementary DNA. Nature. 336:134-139.
4. McRory, J.E., et al. 2004. The CACNA1F gene encodes an L-type calcium channel with unique biophysical properties and tissue distribution. J. Neurosci. 24:1707-1718.

5. Kotturi, M.F., Carlow, D.A., Lee, J.C., Ziltener, H.J., and Jefferies, W.A. 2003. Identification and functional characterization of voltage-dependent calcium channels in T lymphocytes. J. Biol. Chem. 278:46949-46960.

6. Koschak, A., et al. 2003. Cav1.4alpha1 subunits can form slowly inactivating dihydropyridine-sensitive L-type $\mathrm{Ca} 2+$ channels lacking $\mathrm{Ca} 2+$-dependent inactivation. J. Neurosci. 23:6041-6049.

7. Namkung, Y., et al. 2001. Requirement for the L-type $\mathrm{Ca}(2+)$ channel alpha(1D) subunit in postnatal pancreatic beta cell generation. J. Clin. Invest. 108:1015-1022. doi:10.1172/JCI200113310.

8. Platzer, J., et al. 2000. Congenital deafness and sinoatrial node dysfunction in mice lacking class D L-type Ca2+ channels. Cell. 102:89-97.

9. Schulla, V., et al. 2003. Impaired insulin secretion and glucose tolerance in beta cell-selective $\mathrm{Ca}(\mathrm{v}) 1.2$ Ca2+ channel null mice. EMBO J. 22:3844-3854.

10. Hibino, H., et al. 2002. RIM binding proteins (RBPs) couple Rab3-interacting molecules (RIMs) to voltage-gated $\mathrm{Ca}(2+)$ channels. Neuron. 34:411-423.

11. Zhang, Z., et al. 2002. Functional roles of $\mathrm{Ca}(\mathrm{v}) 1.3$ (alpha(1D)) calcium channel in sinoatrial nodes: insight gained using gene-targeted null mutant mice. Circ. Res. 90:981-987.

12. Mangoni, M.E., et al. 2003. Functional role of L-type Cav1.3 Ca2+ channels in cardiac pacemaker activity. Proc. Natl. Acad. Sci. U. S. A. 100:5543-5548.

\title{
Amyloid at the cutting edge: activation of $\alpha$-secretase prevents amyloidogenesis in an Alzheimer disease mouse model
}

\author{
Stefan F. Lichtenthaler and Christian Haass
}

Adolf-Butenandt-Institut, Laboratory for Alzheimer's and Parkinson's Disease Research, Ludwig-Maximilians-Universität, Munich, Germany.

\begin{abstract}
The amyloid $\beta$-peptide (A $\beta$ peptide) is assumed to play a crucial and early role in the pathogenesis of Alzheimer disease. Thus, strategies for a pharmacotherapy aim at reducing $A \beta$ peptide generation, which proteolytically derives from the amyloid precursor protein (APP). The main targets so far have been $\beta$ - and $\gamma$-secretase, the two proteases that cleave APP at the $\mathrm{N}$ - and $\mathrm{C}$-terminus of the $A \beta$ peptide and are thus directly responsible for $A \beta$ peptide generation. A different strategy, namely the activation of $\alpha$-secretase, has barely been investigated for its therapeutic potential. $\alpha$-Secretase cleaves within the $A \beta$ peptide domain and thus precludes $A \beta$ peptide generation. Now, new results demonstrate that activation of $\alpha$-secretase indeed reduces $A \beta$ peptide generation and toxicity in vivo (see the related article beginning on page 1456).
\end{abstract}

\footnotetext{
Nonstandard abbreviations used: a disintegrin and metalloprotease (ADAM); Alzheimer disease (AD); amyloid $\beta$-peptide (A $\beta$ peptide); amyloid precursor protein (APP).

Conflict of interest: The authors have declared that no conflict of interest exists.

Citation for this article:

J. Clin. Invest. 113:1384-1387 (2004).

doi:10.1172/JCI200421746.
}

Numerous laboratories are currently investigating $\beta$ - and $\gamma$-secretase, the two amyloidogenic proteases that cleave the A $\beta$-peptide out of the amyloid precursor protein (APP). The reason is obvious. If we prevent these proteases from working, we will stop the progression of Alzheimer disease (AD). However, a rather old and almost forgotten idea, namely the activation of $\alpha$-secretase, which cuts the amyloid $\beta$-peptide ( $\mathrm{A} \beta$ peptide) into two nonamyloidogenic pieces, has now been reinvestigated. Compelling evidence that this strategy may work is now presented in a study by researchers in Germany and Belgium led by Falk Fahrenholz at the University of Mainz (1).

$\mathrm{AD}$ is the most prevalent neurodegenerative disease, affecting about 20 million people worldwide (for an overview see ref. 2). The amyloid hypothesis of $\mathrm{AD}$, which is now widely accepted, describes the pathogenesis of this disease as a cascade of several steps, from the initial generation of the $A \beta$ peptide to cognitive impairment and neuronal loss (for overviews see refs. 3, 4). Whereas drugs are currently available that may slightly 


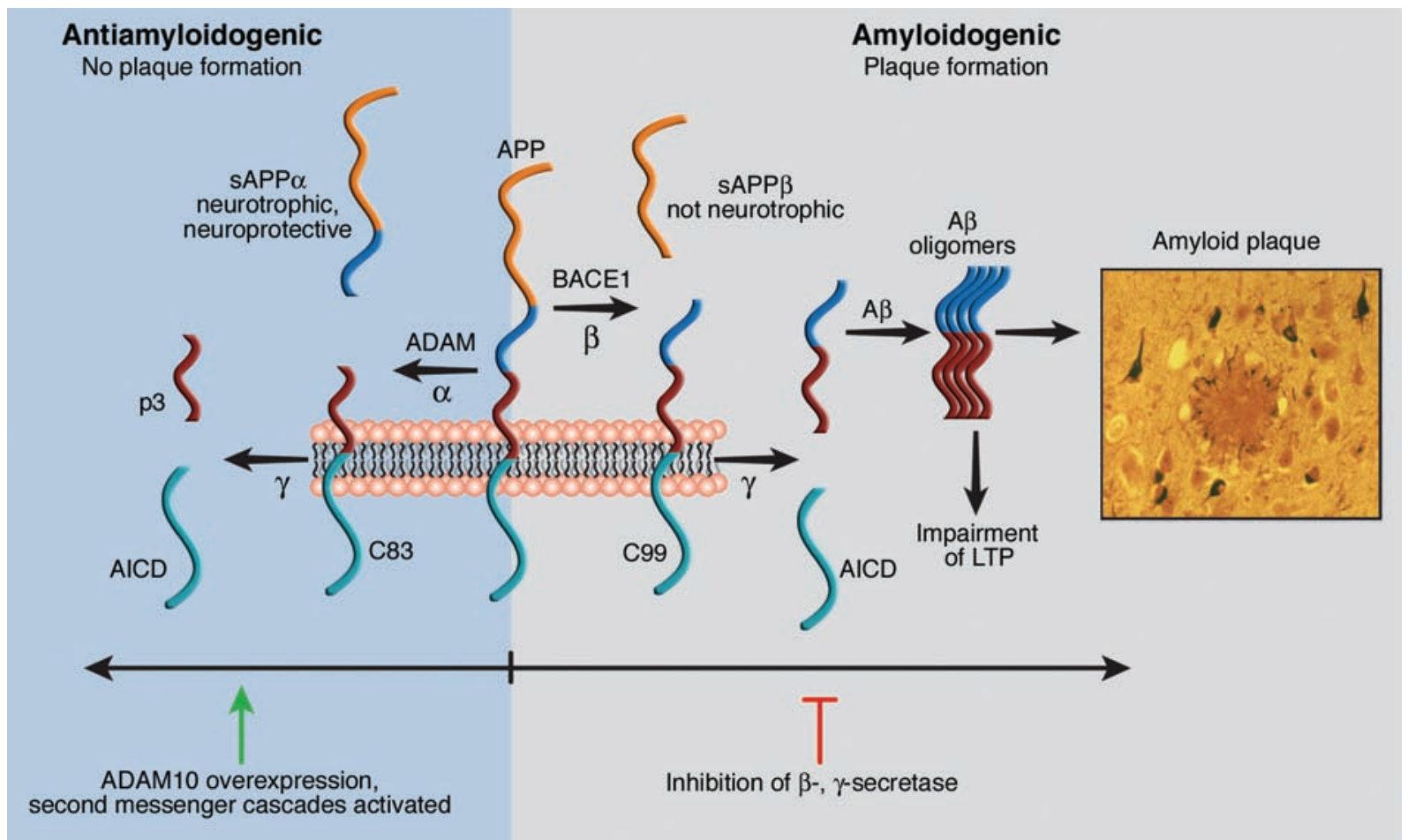

\section{Figure 1}

Proteolytic processing of APP is divided into an amyloidogenic and an antiamyloidogenic pathway. Amyloidogenic pathway: Cleavage of APP by the protease $\beta$-secretase (BACE1) occurs at the $\mathrm{N}$-terminus of the A $\beta$ domain and yields the secreted sAPP $\beta$ as well as a $\mathrm{C}$-terminal fragment of APP of 99 amino acids (C99). C99 is further cleaved within its transmembrane domain by $\gamma$-secretase, leading to the secretion of the A $\beta$ peptide and the generation of the APP intracellular domain (AICD). The A $\beta$ peptide is prone to aggregation. A $\beta$ peptide oligomers are neurotoxic and lead to an impairment of long-term potentiation (LTP). Finally, large amounts of A $\beta$ peptide are deposited in amyloid plaques, which are the characteristic pathological hallmarks of AD. The consecutive cleavage of APP by $\beta$ - and $\gamma$-secretase constitutes the amyloidogenic pathway as it generates A $\beta$. Antiamyloidogenic pathway: Cleavage of APP by $\alpha$-secretase within the A $\beta$ peptide domain yields the neurotrophic and neuroprotective sAPP $\alpha$. The $\alpha$-secretase is a member of the ADAM family of metalloproteases. $\alpha$-Cleavage of APP can be induced upon overexpression of ADAM10 or by the activation of second messenger cascades.

ameliorate late-stage symptoms such as cognitive deficits for a short time, no drugs are on the market that specifically target the cellular mechanisms of the disease, namely the proteolytic generation of the $A \beta$ peptide from APP. APP is a type I membrane protein with unclear biological function. APP undergoes proteolytic processing in two different pathways (Figure 1). One is termed amyloidogenic because it leads to the generation of the $\mathrm{A} \beta$ peptide. The other one is referred to as antiamyloidogenic because it prevents $A \beta$ peptide generation (2).

In the amyloidogenic pathway, APP is first cleaved by the $\beta$-secretase, BACE1, at the $\mathrm{N}$-terminus of the $\mathrm{A} \beta$ domain (5). This cleavage generates the soluble sAPP $\beta$ and a C-terminal fragment, which undergoes a second cleavage by a protease called $\gamma$-secretase. $\gamma$-Secretase cleaves within the transmembrane domain of APP and is a heteromeric protein complex consisting of presenilin, nicastrin, PEN-2, and APH-1 (for a review see ref. 5). The fact that mice deficient in either protease do not generate the $\mathrm{A} \beta$ peptide clearly implicates BACE1 and the $\gamma$-secretase complex as the amyloidogenic proteases in vivo and makes them suitable drug targets for $\mathrm{AD}$ (5).

In contrast, the antiamyloidogenic pathway starts with APP cleavage by $\alpha$-secretase, which cuts within the $A \beta$ domain and thus precludes $A \beta$ peptide generation. Following $\alpha$-cleavage, the C-terminal APP fragment undergoes $\gamma$-cleavage, leading to the generation of the $\mathrm{p} 3$ peptide (6) (Figure 1), which seems to be benign, since it is not found in the amyloid plaques characteristic of AD. $\alpha$-Secretase is a member of the ADAM (a disintegrin and metalloprotease) family of proteases (for a review see ref. 7) and is either ADAM10 (8), ADAM17/TACE (9), or even ADAM9 (10). At present, it is unclear whether only one of them or all three together constitute the physiologically relevant $\alpha$-secretase.
Since the combined action of $\beta$ - and $\gamma$-secretase leads to $A \beta$ peptide generation, the inhibition of their activity is considered to be a highly promising approach to treat $\mathrm{AD}$ and is being pursued by a number of pharmaceutical companies. However, the development of specific $\beta$ and $\gamma$-secretase inhibitors that are able to cross the blood-brain barrier seems to be a particular challenge. BACE1 seems to have a rather unusual large active cleft, which makes the generation of selective inhibitors difficult (11). The suitability of inhibiting $\gamma$-secretase has been called into question by findings that this protease is involved in physiologically highly important signaling mechanisms required for cell fate decisions $(3,12)$. Although these problems may be circumvented at some point, it seems to be increasingly important to search for alternative targets. One such approach may be the facilitation of $\alpha$-secretase cleavage of APP, an idea based on the original findings of Nitsch and 
colleagues (13). Since $\alpha$-secretase cleaves within the $A \beta$ peptide domain, its activation may even have the double advantage of not only precluding the neurotoxic $A \beta$ peptide formation but also generating the putatively neuroprotective sAPP $\alpha(14,15)$. This approach has so far received little attention but is particularly tempting, since BACE1 and $\alpha$-secretase compete for the ectodomain cleavage of APP (16). Thus, it is conceivable to shift APP cleavage from the amyloidogenic $\beta$-secretase to the antiamyloidogenic $\alpha$-secretase cleavage. Indeed, in cultured cells a number of pharmacological agents can stimulate the $\alpha$-secretase cleavage of APP at the expense of $\beta$-cleavage, thereby reducing $A \beta$ peptide generation (for a review see ref. 7). Likewise, overexpression of ADAM proteases such as ADAM10 (8) or ADAM17/TACE (17) also increases APP $\alpha$-cleavage. However, evidence is still lacking that an increased expression or activity of ADAM proteases is antiamyloidogenic in vivo. This is mainly due to the embryonic and perinatal lethality of ADAM10 (18) and ADAM17 (19) knockout mice, respectively, which prevented the functional analysis of these proteases in older mice at an age when amyloid plaques are forming. To circumvent these difficulties, Postina and colleagues (1) chose a different approach to test the potential antiamyloidogenic role of $\alpha$-secretase in vivo. As a natural extension of their original work, which identified ADAM10 as one of the $\alpha$-secretases (8), they chose to overexpress bovine ADAM10 selectively in neurons. Additionally, a dominant-negative ADAM10 mutant was overexpressed, which inhibits the endogenous APP $\alpha$-cleavage in cultured cells (8) and should thus reduce the antiamyloidogenic APP processing in mice. Both transgenic lines were crossed with the $\mathrm{AD}$ mouse model generated in van Leuven's laboratory in Belgium (20). In this model, human APP is overexpressed in neurons. Overexpression of human APP results in enhanced generation of the $A \beta$ peptide, which at the end is deposited in the mouse brain in the same way as in human brains. Furthermore, enhanced $A \beta$ peptide production also results in memory deficits (20). By crossing this AD mouse model with ADAM10 transgenic mice, Postina et al. have now elegantly shown that overexpressed ADAM10 acts as at least one of the $\alpha$-secretases in vivo and thus is indeed antiamyloidogenic (1). ADAM10 overexpression increased sAPP $\alpha$ secretion and reduced $A \beta$ peptide generation. Although the reduction in $\mathrm{A} \beta$ peptide generation was not dramatic, it was sufficient to almost completely prevent amyloid plaque formation in the mouse brain. In contrast, the dominant-negative ADAM10 mutant had the opposite effect of wildtype ADAM10. It inhibited APP $\alpha$-cleavage, slightly increased $A \beta$ peptide generation, and increased both the number and the size of amyloid plaques in the mouse brain. The rather small effects on total $A \beta$ peptide generation as compared to amyloid deposition suggest that relatively minor changes in $\mathrm{A} \beta$ peptide production may be sufficient to induce AD pathology. This provides hope, since secretase modifiers (regardless of whether they are inhibitors of $\beta$ - and $\gamma$-secretase or activators of $\alpha$-secretase) may only be required at rather low dose. Most importantly, the study by Postina and colleagues also provides evidence that overexpression of ADAM10 not only reduces amyloid plaque formation but also alleviates the deficits in spatial learning and synaptic plasticity observed in the control animals (1), suggesting that an activation of $\alpha$-secretase cleavage may also improve cognitive status in humans. On the molecular level, however, it remains unclear whether this beneficial effect is due to the reduced amyloid burden, the increased generation of the neuroprotective sAPP $\alpha$, or both. Moreover, it could even be caused by the increased secretion of other protein substrates of ADAM10. However, the mice expressing ADAM10 that were initially generated (before being crossed to the APPexpressing mice) did not show any obvious phenotypic changes compared to control animals, which is good news, given that ADAM10 is also involved in the cleavage of membrane proteins other than APP, such as Notch (18), EGF, and $\beta$-cellulin (21).

To fully evaluate the therapeutic potential of activation of $\alpha$-secretase, additional studies are needed. In contrast to the transgenic mice overexpressing ADAM10 from shortly after birth, humans would not be treated until later in life. Thus, it will be important to use mice with an inducible ADAM10 expression to address the question whether ADAM10 still lowers $A \beta$ peptide generation and rescues memory deficits when overexpressed later in life, when the first plaques already have formed. A direct transfer of the murine results into patients would require a gene therapy approach to overexpress ADAM10 in neurons. Since this may not be easily feasible in humans, it is of utmost importance to explore other ways to increase the expres- sion and activity of ADAM proteases in vivo. From tissue culture studies it is well known that the ADAM cleavage of APP can be stimulated through an activation of second messenger cascades (for an overview see ref. 7). However, at present little is known about the underlying cellular pathways and mechanisms. Therefore, the identification of regulatory genes and chemical compounds that selectively affect ADAM-protease activity may lead to new drug targets and new possibilities for pharmacological intervention in AD. However, one has to keep in mind that activation of the second messenger cascades is a rather nonselective approach which again may result in unwanted side effects. The work by Fahrenholz and colleagues is a major step forward in exploring the therapeutic potential of $\alpha$-secretase targeting.

Address correspondence to: Stefan F. Lichtenthaler or Christian Haass, Adolf-Butenandt-Institut, Laboratory for Alzheimer's and Parkinson's Disease Research, LudwigMaximilians-Universität, Schillerstrasse 44, 80336 Munich, Germany. (S.F.L.) Phone: 49-89-5996-453; Fax: 49-5996-415; E-mail: Stefan.Lichtenthaler@med.uni-muenchen. de. (C.H.) Phone: 49-89-5996-471; Fax: 495996-415; E-mail: Christian.Haass@med. uni-muenchen.de.

1. Postina, R., et al. 2004. A disintegrin-metalloproteinase prevents amyloid plaque formation and hippocampal defects in an Alzheimer disease mouse model. J. Clin. Invest. 113:1456-1464. doi:10.1172/JCI200420864.

2. Selkoe, D.J., and Schenk, D. 2003. Alzheimer's disease: molecular understanding predicts myloidbased therapeutics. Annu. Rev. Pharmacol. Toxicol. 43:545-584.

3. Citron, M. 2004. beta-Secretase inhibition for the treatment of Alzheimer's disease - promise and challenge. Trends Pharmacol. Sci. 25:92-97.

4. Hardy, J., and Selkoe, D.J. 2002. The amyloid hypothesis of Alzheimer's disease: progress and problems on the road to therapeutics. Science. 297:353-356.

5. Haass, C. 2004. Take five-BACE and the gammasecretase quartet conduct Alzheimer's amyloid beta-peptide generation. EMBOJ. 23:483-488.

6. Haass, C., et al. 1993. b-Amyloid peptide and a $3-\mathrm{kDa}$ fragment are derived by distinct cellular mechanisms. J. Biol. Chem. 268:3021-3024.

7. Allinson, T.M., Parkin, E.T., Turner, A.J., and Hooper, N.M. 2003. ADAMs family members as amyloid precursor protein alpha-secretases. J. Neurosci. Res. 74:342-352.

8. Lammich, S., et al. 1999. Constitutive and regulated alpha-secretase cleavage of Alzheimer's amyloid precursor protein by a disintegrin metalloprotease. Proc. Natl. Acad. Sci. U. S. A. 96:3922-3927.

9. Buxbaum, J.D., et al. 1998. Evidence that tumor necrosis factor alpha converting enzyme is involved in regulated alpha-secretase cleavage of the Alzheimer amyloid protein precursor. J. Biol. 
Chem. 273:27765-27767.

10. Koike, H., et al. 1999. Membrane-anchored metalloprotease MDC9 has an alpha-secretase activity responsible for processing the amyloid precursor protein. Biochem. J. 343:371-375.

11. Hong, L., et al. 2000. Structure of the protease domain of memapsin 2 (beta-secretase) complexed with inhibitor. Science. 290:150-153.

12. Selkoe, D., and Kopan, R. 2003. Notch and Presenilin: regulated intramembrane proteolysis links development and degeneration. Annu. Rev. Neurosci. 26:565-597.

13. Nitsch, R.M., Slack, B.E., Wurtman, R.J., and Growdon, J.H. 1992. Release of Alzheimer amyloid precursor derivatives stimulated by activation of muscarinic acetylcholine receptors. Science. 258:304-307.
14. Furukawa, K, et al. 1996. Increased activity-regulating and neuroprotective efficacy of alphasecretase-derived secreted amyloid precursor protein conferred by a C- terminal heparin-binding domain. J. Neurochem. 67:1882-1896.

15. Meziane, H., et al. 1998. Memory-enhancing effects of secreted forms of the beta-amyloid precursor protein in normal and amnestic mice. Proc. Natl. Acad. Sci. U. S. A. 95:12683-12688.

16. Skovronsky, D.M., Moore, D.B., Milla, M.E., Doms, R.W., and Lee, V.M. 2000. Protein kinase C-dependent alpha-secretase competes with betasecretase for cleavage of amyloid-beta precursor protein in the trans-golgi network. J. Biol. Chem. 275:2568-2575.

17. Slack, B.E., Ma, L.K., and Seah, C.C. 2001. Constitutive shedding of the amyloid precursor protein ectodomain is up-regulated by tumour necrosis factor-alpha converting enzyme. Biochem. J. 357:787-794.

18. Hartmann, D., et al. 2002. The disintegrin/ metalloprotease ADAM 10 is essential for Notch signalling but not for alpha-secretase activity in fibroblasts. Hum. Mol. Genet. 11:2615-2624.

19. Peschon, J.J., et al. 1998. An essential role for ectodomain shedding in mammalian development. Science. 282:1281-1284

20. Moechars, D., et al. 1999. Early phenotypic changes in transgenic mice that overexpress different mutants of amyloid precursor protein in brain. J. Biol. Chem. 274:6483-6492.

21. Sahin, U, et al. 2004. Distinct roles for ADAM10 and ADAM17 in ectodomain shedding of six EGFR ligands. J. Cell. Biol. 164:769-779.

\section{Sepsis: avoiding its deadly toll}

Thomas Decker

Max F. Perutz Laboratories, University Departments at the Vienna Biocenter, Department of Microbiology and Genetics, University of Vienna, Vienna, Austria.

\begin{abstract}
Systemic bacterial infection may culminate in a frequently fatal septic shock syndrome. The underlying pathology is the result of an uncontrolled inflammatory response, stimulated by the pathogen and its products. Tolllike receptors (TLRs) are critically involved in sensing bacteria and, in the case of sepsis, stimulate a pathogenic response by the innate immune system. A new study reports a successful attempt to inhibit systemic inflammation in mice by disrupting the formation of complexes between Grampositive bacteria and their cognate receptor, TLR2 (see the related article beginning on page 1473).
\end{abstract}

Sepsis results from the inability of the immune system to limit bacterial spread during an ongoing infection. Massive bacterial load overrides the inhibitory mechanisms controlling inflammation. While normally helping to eradicate pathogens from a local infection of peripheral tissues, inflammation during sepsis develops into a systemic syndrome with multiple manifestations such as tissue injury, increased vascular permeability, and, ultimately, multi-organ failure and shock (1). Mortality rates of septic patients are high (up to $70 \%$ ), and the costs of treatment have been calculated to amount to more than $\$ 15$ billion per year in the US alone (2). Since antibiotics carry the risk of promoting the release of bacterial products and thus to exacerbate the shock syndrome during sepsis, the development of

Nonstandard abbreviations used: high-mobility group box 1 protein (Hmgb1); pathogen-associated molecular pattern (PAMP); toll-like receptor (TLR). Conflict of interest: The author has declared that no conflict of interest exists.

Citation for this article:

J. Clin. Invest. 113:1387-1389 (2004).

doi:10.1172/JCI200421819. alternative treatments and the improvement of current regimens for treating septic patients are of high priority.

\section{The septic shock syndrome and its mediators}

The list of suitable targets for interruption of the inflammatory cascade begins with the receptors involved in the binding and uptake of bacteria and their products by cells of the innate immune system (phagocytes, dendritic cells, vascular endothelial cells). It continues with the many proinflammatory molecules produced by the innate immune system during infection, cytokines and/or chemokines like TNF- $\alpha$, IL-1, and IL-8, lipid mediators, oxygen radicals, and tissue-damaging enzymes. Components of the activated complement and coagulation systems also promote inflammation and are further candidate targets for inhibitory drugs (Figure 1). Several novel therapeutic approaches have been tested in animal models of septic shock and also in clinical trials (3). Activated protein C, an inhibitor of clotting factors, reduces monocyte synthesis of proinflammatory cytokines and the interaction of phagocytes with the vascular endothelium and has been approved for clinical use (reviewed in ref. 3 ). The therapeutic approach reported in this issue of the JCI by Meng and colleagues explores the possibility of interrupting an initial step of septic inflammation, the interaction of Gram-positive bacteria or their cell wall components with toll-like receptor 2 (TLR2) (4).

\section{TLRs in innate immune responses to pathogens}

TLRs belong to the group of germlineencoded, nonclonal receptors, which is functionally defined by the ability to discriminate self from pathogen-associated molecular patterns (PAMPs) (5). Ten members of the TLR family in humans and 11 in rodents allow cells of the innate immune system to respond with appropriate intracellular signals to the presence of all groups of microorganisms (6, $7)$. TLR signaling stimulates cell-autonomous antimicrobial defense systems as well as the secretion of immunoregulatory substances, including many of the proinflammatory molecules listed above. Associations between the susceptibility to pathogens and defective or absent TLRs have been established by genetic linkage with inactivating mutations in humans (8), and by targeted disruption of th loci in mice (6). Further examples of immunological benefits resulting from TLR stimulation have been provided by the antiviral imidazoquinolines and guanosine analogues that exert their effects via TLRs 